\title{
Management of major depressive disorder with psychotic features
}

Rachel Barr, PharmD, BCPP, Ben Miskle, PharmD, and Christopher Thomas, PharmD, BCPS, BCPP

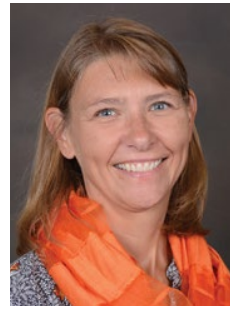

Vicki L. Ellingrod, PharmD, FCCP

Department Editor

Sawy Psychopharmacology is produced in partnership with the College of Psychiatric and Neurologic Pharmacists cpnp.org mhc.cpnp.org (journal)
$\mathrm{M}$ rs. C, age 56, has a history of major depressive disorder (MDD). She has been stable for 5 years without medication. Six months ago, she presented to you, along with her son, seeking help. She reported that she had been experiencing insomnia, fatigue, and was not engaging in hobbies. Her son told you that his mother had lost weight and had been avoiding family dinners. Mrs. C reported recurrent thoughts of dying and heard voices vividly telling her that she was a burden and that her family would be better off without her. However, there was no imminent danger of self-harm. At that appointment, you initiated sertraline, $50 \mathrm{mg} / \mathrm{d}$ titrated to $100 \mathrm{mg} / \mathrm{d}$, and olanzapine, $5 \mathrm{mg} / \mathrm{d}$.

Since that time, Mrs. C has followed up with you monthly with good response to the medications. Currently, she states her depression is much improved, and she denies hearing voices for approximately 5 months.

Dr. Barr is a Clinical Psychiatric Pharmacist, Eastern Oklahoma VA Healthcare System, Tulsa, Oklahoma. Dr. Miskle is a Clinica Psychiatric Pharmacist, University of lowa Hospitals and Clinics, lowa and Clinical Assistant Professor, University of lowa College of Pharmacy, lowa City, lowa. Dr. Thomas is Director, PGY-1 and PGY-2 Residency Programs, Clinical Pharmacy Specialist in Psychiatry, Chillicothe VA Medical Center, Chillicothe, Ohio, and Clinical Associate Professor of Pharmacology, Ohio University College of Osteopathic Medicine, Athens, Ohio.

\section{Disclosures}

The contents of this article do not represent the views of the US Department of Veterans Affairs or the US Government. This material is the result of work supported with resources and the use of facilities at the Chillicothe Veterans Affairs Medical Center in Chillicothe, Ohio The case presented in this article is fictional and does not represent a specific case or person(s).

The authors report no financial relationships with any companies whose products are mentioned in this article, or with manufacturers of competing products.

doi: $10.12788 /$ cp.0092
Based on her presentation and response, what do the data suggest about her length of treatment, and when should you consider tapering the antipsychotic medication?

In DSM-5, MDD with psychotic features is a severe subtype of MDD that is defined as a major depressive episode characterized by delusions and/or hallucinations. ${ }^{1}$ In the general population, the lifetime prevalence of this disorder varies from $0.35 \%$ to $1 \%$, and the rate is higher in older patients. ${ }^{2}$ Risk factors include female gender, family history, and concomitant bipolar disorder. ${ }^{2}$

\section{Practice Points}

- In patients experiencing major depressive disorder (MDD) with psychotic features, consider electroconvulsive therapy or an antidepressant in combination with an antipsychotic.

- Any antipsychotic can be considered for the treatment of MDD with psychotic features because there are no head-to-head trials. However, because second-generation antipsychotics have a lower risk of adverse effects, they should be preferred to first-generation antipsychotics.

-When prescribing combination therapy, continue treatment for at least 9 months to reduce the risk of relapse, and consider reducing the dose of the antipsychotic medication after 1 year of treatment.

-When stopping antipsychotic therapy, implement a slow and gradual taper to allow for assessment of symptom recurrence. 
Epidemiologic studies have shown that psychotic features can occur in $15 \%$ to $20 \%$ of patients with MDD. The psychotic features that occur during these episodes are delusions and hallucinations. ${ }^{1}$ These features can be either mood-congruent (related to the depressive themes of worthlessness or guilt) or mood-incongruent (ie, unrelated to depressive themes). ${ }^{1}$

\section{Treatment options: ECT or pharmacotherapy}

Guidelines from the American Psychiatric Association $^{3}$ and the National Institute for Clinical Excellence ${ }^{4}$ recommend treating depression with psychosis with electroconvulsive therapy (ECT) or with combined antidepressant and antipsychotic medications as first-line options. The Texas Medication Algorithm Project (TMAP) Algorithm for MDD, ${ }^{5}$ which closely focuses on treatment of MDD with psychotic features, can be used for treatment decisions (see Related Resources, page 32).

Electroconvulsive therapy is known to be efficacious in treating patients with MDD with psychotic features and should be considered as a treatment option. However, medication therapy is often chosen as the initial treatment due to the limitations of ECT, including accessibility, cost, and patient preference. However, in certain cases, ECT is the preferred option because it can provide rapid and significant improvement in patients with severe psychosis, suicidality, or risk of imminent harm.

Pharmacotherapy for the treatment of MDD with psychotic features should consist of a combination of an antidepressant and antipsychotic medication. This combination has been shown to be more effective than either agent alone. Some combinations have been studied specifically for MDD with psychosis. The Study of the Pharmacotherapy of Psychotic Depression (STOP-PD), a 12-week, double-blind, randomized controlled trial, found that the combination of sertraline and olanzapine was efficacious and superior to monotherapy with olanzapine in an acute setting. ${ }^{6}$ In another study, the combination of olanzapine and fluoxetine was also found to be superior to olanzapine monotherapy in reducing Hamilton Depression Rating Scale (HAM-D) scores. ${ }^{7}$ Quetiapine, when used in combination with venlafaxine, was found to be superior to venlafaxine monotherapy in response. ${ }^{8}$ Lastly, amitriptyline in combination with either haloperidol or perphenazine has been shown to be superior to monotherapy. ${ }^{9,10}$ However, no medications are specifically FDA-approved for the indication of depression with psychotic features. Because none of these agents have been compared in head-to-head trials, any combination of antidepressant and antipsychotic medication can be used. Due to the greater risk of adverse effects with first-generation antipsychotics (FGAs), such as extrapyramidal symptoms (EPS), second-generation antipsychotics (SGAs) should be trialed first.

\section{How long should treatment last?}

The optimal timeline for treating patients with MDD with psychotic features is unknown. According to the TMAP algorithm and expert opinion, the continuation phase of pharmacotherapy should include treatment for at least 4 months with an antipsychotic medication and at least 2 years to lifetime treatment with an antidepressant. ${ }^{5}$ The STOP-PD II study, which was a continuation of the 12-week STOP-PD study, examined antipsychotic duration to determine the effects of continuing olanzapine once an episode of psychotic depression had responded to olanzapine and sertraline. ${ }^{11}$ Patients who had achieved remission after receiving olanzapine and sertraline were randomized to continue to receive this combination or to receive sertraline plus placebo for 36 weeks. The primary outcome was relapse, which was broadly defined as 1 of the following ${ }^{11}$ :

- a Structured Clinical Interview for the DSM (SCID)-rated assessment that revealed the patient had enough symptoms to meet criteria for a DSM-IV major depressive episode

\section{Clinical Point \\ ECT can provide rapid and significant improvement in patients with severe psychosis, suicidality, or risk of imminent} harm

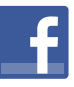

Discuss this article at www.facebook.com/ MDedgePsychiatry 


\section{Clinical Point}

When prescribing
combination
therapy, continue
treatment for at least
9 months to reduce
the risk of relapse

\section{Related Resources}

- Texas Medication Algorithm Project. Algorithm for the treatment of major depressive disorder with psychotic features. https://chsciowa.org/sites/chsciowa.org/files/ resource/files/9_-_depression_med_algorithm supplement.pdf

- Dold M, Bartova L, Kautzky A, et al. Psychotic features in patients with major depressive disorder: a report from the European Group for the Study of Resistant Depression. J Clin Psychiatry. 2019;80(1):17m12090. doi: 10.4088/ JCP. $17 \mathrm{~m} 12090$

- Flint AJ, Meyers BS, Rothschild AJ, et al. Effect of continuing olanzapine vs placebo on relapse among patients with psychotic depression in remission: the STOP-PD II randomized clinical trial. JAMA. 2019;322(7): 622-631.

\section{Drug Brand Names}

\section{Amitriptyline • Elavil, Endep}

Fluoxetine - Prozac

Haloperidol $\cdot$ Haldol

Olanzapine - Zyprexa

- a 17-item HAM-D scoren of $\geq 18$

- SCID-rated psychosis

- other significant clinical worsening, defined as having a suicide plan or attempting suicide, developing SCID-rated symptoms of mania or hypomania, or being hospitalized in a psychiatric unit.

Compared with sertraline plus placebo, continuing sertraline plus olanzapine reduced the risk of relapse over 36 weeks (hazard ratio, 0.25; 95\% confidence interval, 0.13 to $0.48 ; P<.001) .{ }^{11}$ However, as expected, the incidence of adverse effects such as weight gain and parkinsonism was higher in the olanzapine group. Therefore, it is important to consider the potential long-term adverse effects of continuing antipsychotic medications. The STOP-PD II trial showed benefit in continuing antipsychotic therapy over 36 weeks, but did not answer the question of how long to continue antipsychotic therapy.

\section{Weighing the evidence}

Electroconvulsive therapy is considered a first-line treatment option for MDD with psychotic features; however, because of limitations associated with this approach, antidepressants plus antipsychotics are often utilized as an initial treatment. Essentially, any antipsychotic agent can be prescribed in conjunction with an antidepressant, but due to the greater risk of adverse effects associated with FGAs, SGAs should be trialed first. The results of the STOP-PD 6 and STOP-PD II ${ }^{11}$ studies have shown that once a patient responds to an antidepressant and antipsychotic, combination therapy needs to continue for at least 9 months to reduce the risk of relapse. Thereafter, reducing the dose of the antipsychotic can be considered after 1 year of treatment; however, no data exist about which agent and tapering schedule to consider. Because no optimal duration has been fully established, consider a slow and gradual taper when stopping antipsychotic therapy to allow for assessment of recurring symptoms.

\section{CASE CONTINUED}

Based on the results of the STOP-PD and STOP-PD II trials, Mrs. C should be continued on sertraline plus olanzapine for at least another 3 to 6 months before an olanzapine taper should be considered. At that time, the risks and benefits of a taper vs continuing therapy should be considered. Given her history of MDD and the severity of this most recent episode, sertraline therapy should be continued for at least 2 years, and possibly indefinitely.

\section{References}

1. Diagnostic and statistical manual of mental disorders, 5 th ed. American Psychiatric Association; 2013.

2. Jääskeläinen E, Juola T, Korpela H, et al. Epidemiology of psychotic depression - systematic review and meta-analysis. Psychol Med. 2018;48(6):905-918.

3. American Psychiatric Association. Practice guideline for the treatment of patients with major depressive disorder (revision). Am J Psychiatry. 2000;157(4)(suppl):1-45.

4. National Institute for Clinical Excellence. Depression in adults: recognition and management: clinical guideline [CG90]. National Institute for Health and Clinical Excellence. Published October 28, 2009. Accessed January 12, 2021. https://www.nice.org.uk/guidance/cg90

5. Crimson ML, Trivedi M, Pigott TA, et al. The Texas Medication Algorithm Project: report of the Texas Consensus Conference Panel on medication treatment of major depressive disorder. J Clin Psychiatry. 1999;60(3): 142-156.

6. Meyers BS, Flint AJ, Rothschild AJ, et al. A double-blind randomized controlled trial of olanzapine plus sertraline vs 
olanzapine plus placebo for psychotic depression: the study of pharmacotherapy for psychotic depression -- the STOPPD study. Arch Gen Psychiatry. 2009;66(8):838-847.

7. Rothschild AJ, Williamson DJ, Tohen MF, et al. A doubleblind, randomized study of olanzapine and olanzapine/ fluoxetine combination for major depression with psychotic features. J Clin Psychopharmacol. 2004;24(4):365-373.

8. Wijkstra J, Burger H, van den Broek WW, et al. Treatment of unipolar psychotic depression: a randomized, doubleblind study comparing imipramine, venlafaxine, and venlafaxine plus quetiapine. Acta Psychiatr Scand. 2010;21(3):190-200.
9. Muller-Siecheneder F, Muller M, Hillert A, et al. Risperidone versus haloperidol and amitriptyline in the treatment of patients with a combined psychotic and depressive syndrome. J Clin Psychopharm. 1998;18(2):111-120.

10. Spiker DG, Weiss JC, Dealy RS, et al. The pharmacological treatment of delusional depression. Am J Psychiatry. 1985; 142(4):430-436.

11. Flint AJ, Meyers BS, Rothschild AJ, et al. Effect of continuing olanzapine vs placebo on relapse among patients with psychotic depression in remission: the STOP-PD II randomized clinical trial. JAMA. 2019;322(7):622-631.
Clinical Point

Consider stopping the antipsychotic after 1 year, but use a slow and gradual taper 